\title{
Acute Preoperative Infarcts and Poor Cerebrovascular Reserve Are Independent Risk Factors for Severe Ischemic Complications following Direct Extracranial-Intracranial Bypass for Moyamoya Disease
}

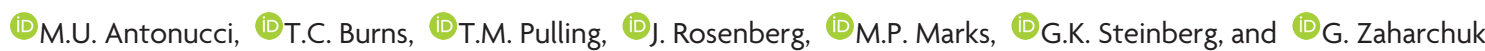

\begin{abstract}
BACKGROUND AND PURPOSE: Severe ischemic changes are a rare but devastating complication following direct superficial temporal artery to MCA bypass in patients with Moyamoya disease. This study was undertaken to determine whether preoperative MR imaging and/or cerebrovascular reserve assessment by using reference standard stable xenon-enhanced CT could predict such complications.
\end{abstract}

MATERIALS AND METHODS: Among all adult patients undergoing direct bypass at our institution between 2005 and 2010 who received a clinically interpretable xenon-enhanced CT examination, we identified index cases (patients with $>15$-mL postoperative infarcts) and control cases (patients without postoperative infarcts and without transient or permanent ischemic symptoms). Differences between groups were evaluated by using the Mann-Whitney $U$ test. Univariate and multivariate generalized linear model regression was used to test predictors of postoperative infarct.

RESULTS: Six index cases were identified and compared with 25 controls. Infarct size in the index cases was $95 \pm 55 \mathrm{~mL}$. Four of 6 index cases (67\%), but no control patients, had preoperative acute infarcts. Baseline CBF was similar, but cerebrovascular reserve was significantly lower in the index cases compared with control cases. For example, in the anterior circulation, median cerebrovascular reserve was $-0.4 \%$ (range, $-38.0 \%-16.6 \%$ ) in index versus $26.3 \%$ (range, $-8.2 \%-60.5 \%$ ) in control patients $(P=.003)$. Multivariate analysis demonstrated that the presence of a small preoperative infarct (regardless of location) and impaired cerebrovascular reserve were independent, significant predictors of severe postoperative ischemic injury.

CONCLUSIONS: Acute infarcts and impaired cerebrovascular reserve on preoperative imaging are independent risk factors for severe ischemic complications following superficial temporal artery to MCA bypass in Moyamoya disease.

ABBREVIATIONS: CVR = cerebrovascular reserve; STA-MCA = superficial temporal artery to middle cerebral artery; $\mathrm{XeCT}=$ xenon-enhanced CT

M oyamoya disease is a cerebrovascular disorder characterized by steno-occlusion of the supraclinoid internal carotid, proximal anterior, and middle cerebral arteries. The entity is discernible on cerebral angiography, on which these findings are frequently associated with a characteristic collateral network at the base of the brain. ${ }^{1,2}$ In addition, leptomeningeal anastomoses

\section{Received April 27, 2015; accepted after revision July 3.}

From the Department of Radiology (M.U.A.), Medical University of South Carolina, Charleston, South Carolina; and Departments of Neurosurgery (T.C.B., G.K.S.) and Radiology (T.M.P., J.R., M.P.M., G.Z.), Stanford University and Stanford University Medical Center, Stanford, California.

M.U. Antonucci and T.C. Burns contributed equally.

This work was supported by National Institutes of Health 1R01-NS066506.

Please address correspondence to Greg Zaharchuk, MD, PhD, Department of Radiology, Stanford University, 1201 Welch Rd, mail code 5488, Stanford,

CA 94305-5488; e-mail: gregz@stanford.edu

- Indicates open access to non-subscribers at www.ajnr.org

http://dx.doi.org/10.3174/ajnr.A4535 from the posterior circulation and transdural anastomoses from the external carotid artery can develop to preserve cerebral perfusion. ${ }^{3-5}$ Patients present with transient ischemic attacks, ischemic strokes, intracranial hemorrhage, seizures, and/or headaches. ${ }^{6,7}$ Its natural history, while variable, is typically relentlessly progressive.

Surgical revascularization has emerged as an effective treatment to prevent future ischemic episodes. ${ }^{1,3,8}$ Revascularization procedures can be characterized as either direct (eg, superficial temporal artery to MCA [STA-MCA] bypass), indirect (eg, encephaloduroarteriosynangiosis), or combined. ${ }^{3,9}$ While direct bypass shows no benefit over medical therapy for treating atherosclerotic disease, ${ }^{10,11}$ it continues to be successfully used for Moyamoya disease. The overwhelming majority of these procedures are uncomplicated, with low perioperative morbidity. ${ }^{9}$ Rarely, ischemic complications can lead to disability or death. In the present study, we tested whether preoper- 
ative imaging and cerebrovascular reserve (CVR) assessment can identify patients at higher risk of severe early ischemic postoperative complications.

\section{MATERIALS AND METHODS Patient Population}

We performed a retrospective case-control study, comprising populations drawn from the 279 adult patients with Moyamoya disease who underwent 438 STA-MCA bypasses at Stanford University between 2005 and 2010. These patients underwent a standardized, extensive preoperative work-up, including cerebral angiography, MR imaging, and CVR assessment. All patients were participants in an ongoing Moyamoya disease study, for which institutional review board approval and informed consent were obtained. All procedures were in accordance with institutional guidelines.

Our analysis focused on 2 subgroups of the full cohort. Given our interest in CVR, patients were eligible for inclusion if they had a diagnostic pre- and postacetazolamide xenon-enhanced CT (XeCT) study as part of their preoperative assessment. The index cases are patients with severe ischemic complications. We defined "severe ischemia" as a postoperative infarct of $>15 \mathrm{~mL}$ on diffusion-weighted imaging coupled with new symptoms within 1 week of surgery. The control cases are patients with no new postoperative symptoms, even if transient, and no new DWI lesion of any size on postoperative MR imaging.

\section{Imaging}

All patients had preoperative MR imaging and XeCT imaging within 1 month of surgery. "Acute infarct" was defined as high DWI signal, confirmed on apparent diffusion coefficient maps to exclude T2 shinethrough. Postoperative MR imaging with DWI was performed in all patients within 1 week of the operation. Infarct volume was calculated by using automated software (RApid processing of Perfusion and Diffusion; iSchemaView, Stanford, California). ${ }^{12}$

Cerebral hemodynamic assessment was performed by using pre- and postacetazolamide XeCT CBF imaging. The study was performed by using a LightSpeed 8-detector scanner (GE Healthcare, Milwaukee, Wisconsin) integrated with a stable xenon-enhancer system (Enhancer 3000; Diversified Diagnostic Products, Houston, Texas). Following an initial noncontrast CT, 4 contiguous 10 -mm sections, aligned with the orbitomeatal axis and beginning at the level of the basal ganglia, were obtained. Images were acquired at 45 -second intervals during which the patient breathed a mixture containing $27 \%$ stable xenon gas. CBF in units of milliliter/100 g/min was calculated by using the Kety-Schmidt method as implemented by the manufacturer's (Diversified Diagnostic Products) software. This software also permits the brain to be segmented into peripheral mixed cortical ROIs in each section (Fig 1). The regions were combined on the basis of anatomic territories to generate values for 4 vascular territories: right and left, anterior (anterior and middle cerebral artery territories) and posterior regions. ${ }^{13,14}$

Cerebrovascular reserve assessment was performed by using CBF values obtained before (pre) and 20 minutes following

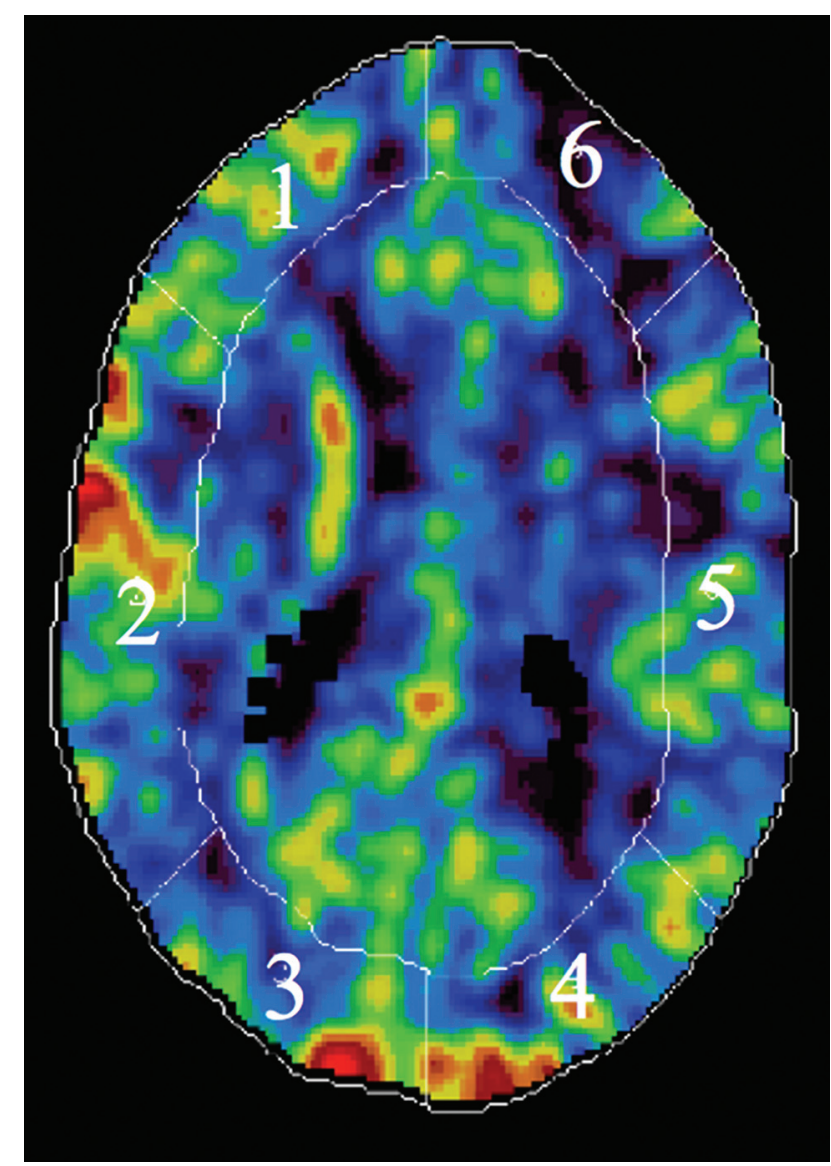

FIG 1. A representative xenon-enhanced CT study with ROIs for analysis. At each of 4 contiguous 10-mm sections starting at the level of the basal ganglia, the brain is segmented into 6 peripheral mixed cortical ROIs (a single section is shown here for clarity). For the anterior circulation CBF levels reported, the weighted average of regions 1 and 2 (right hemisphere) and 5 and 6 (left hemisphere) for all 4 levels was determined; likewise, the same method by using regions 3 and 4 was used to determine the right and left posterior circulation CBF values.

(post) $1 \mathrm{~g}$ of acetazolamide administered intravenously. CVR is defined as

$$
C V R=\frac{C B F_{\text {post }}-C B F_{\text {pre }}}{C B F_{\text {pre }}}
$$

and expressed as percentage change. In the normal brain, acetazolamide will produce a $30 \%-60 \%$ CBF increase. ${ }^{15}$ However, in Moyamoya disease, areas of the brain under ischemic stress may demonstrate an insufficient response to vasodilatory stimuli or even a paradoxically decreased postacetazolamide CBF, known as the "steal phenomenon."

\section{STA-MCA Bypass}

The surgical technique of the STA-MCA bypass has been described in detail previously. ${ }^{16-18}$ In brief, the frontal or parietal branch of the STA is anastomosed end-to-side with an M4 MCA branch. Graft patency is confirmed intraoperatively, and strict blood pressure control is maintained throughout the operation. Care is taken to avoid significant hypotensive periods intraoperatively. Patients are maintained on aspirin and kept well-hydrated postoperatively with close hemodynamic monitoring. ${ }^{16-18}$ In pa- 
Table 1: Demographic, clinical, and imaging characteristics of index and control cases ${ }^{a}$

\begin{tabular}{lccc}
\hline \multicolumn{1}{c}{ Factor } & Index $(\boldsymbol{n}=\mathbf{6})$ & Control $(\boldsymbol{n}=\mathbf{2 5})$ & Group Effect $(\boldsymbol{P}$ Value) \\
\hline Age $(\mathrm{yr})$ & $39(27-67)$ & $35(18-59)$ & .39 \\
Sex $(\%$ female) & 67 & 68 & .67 \\
Anterior territory CBF baseline $(\mathrm{mL} / 100 \mathrm{~g} / \mathrm{min})$ & $36.6(33.3-57.9)$ & $50.2(31.2-62.9)$ & .14 \\
Anterior territory CBF post-ACZ (mL/100 g/min) & $34.3(23.4-64.5)$ & $63.7(39.0-74.3)$ & .007 \\
Anterior change CBF (CVR) $(\%)$ & $-0.4(-38.7-16.6)$ & $26.3(-8.2-60.5)$ & .003 \\
Posterior territory CBF baseline $(\mathrm{mL} / 100 \mathrm{~g} / \mathrm{min})$ & $41.7(33.4-48.8)$ & $38.8(27.1-54.5)$ & .36 \\
Posterior territory CBF post-ACZ (mL/100 g/min) & $54.2(31.7-64.7)$ & $55.6(38.2-72.2)$ & .39 \\
Posterior change CBF (CVR) $(\%)$ & $31.9(-19.1-55.4)$ & $39.1(0.9-117.7)$ & .16 \\
Volume of acute infarcts following surgery (mL) & $95.0(17.0-181.0)$ & 0 & $\mathrm{NA}$ \\
Patients with acute (DWI+) infarcts on preop MRI (\%) & $4(66.7 \%)$ & $0(0 \%)$ & $\mathrm{NA}$ \\
\hline
\end{tabular}

Note:-ACZ indicates acetazolamide; preop, preoperative; NA, not applicable; DWI+, DWI with positive findings.

${ }^{a}$ Values are median (range) where applicable.

tients with bilateral disease, staged unilateral operations are typically performed 1 week apart.

\section{Statistical Analysis}

Differences between index and control groups were assessed by using the Mann-Whitney $U$ test. Sex correlation was evaluated by using the $\chi^{2}$ test. Because the presence of preoperative infarcts, CVR, and postoperative infarcts may vary between hemispheres, analyses were further performed in each right and left anterior and right and left posterior territory. Because several patients underwent bilateral bypasses, assessment of these variables using an ipsilateral/contralateral convention was not possible and a right/ left distinction was used. When practical, measured parameters such as CBF and CVR were combined to produce anterior and posterior values. Predictors of preoperative augmentation were tested with univariate Wilcoxon rank sum tests and multivariate mixed-effects linear regression with patient as the random effect. Predictors of postoperative infarct were tested with univariate and multivariate generalized linear model regression adjusted for clustering within patient. Because of the low frequency of strokes, a loglog link was used instead of the usual logit link. For univariate and multivariate regression, we dichotomized CVR to a greater than or less than $10 \%$ CBF increase ${ }^{19}$ and $<0 \%$ increase (cerebrovascular steal). All statistical analyses were performed by using STATA 13.1 (StataCorp, College Station, Texas).

\section{RESULTS}

Six index cases (with 3 patients undergoing bilateral surgeries for 9 bypassed hemispheres) and 25 control cases (with 36 bypassed hemispheres) were identified. Demographic data and preoperative imaging characteristics for each group are presented in Table 1.

\section{DWI Analysis}

The mean size of the postoperative DWI lesions in the index group was large $(95 \pm 55 \mathrm{~mL})$. Figure 2 shows representative MR images from each index patient demonstrating the infarct patterns, which were not restricted to the bypassed territory and often involved both cortical and subcortical structures. On preoperative MR imaging, 4 of the 6 index cases (67\%) had small acute infarcts. In 3 patients, there were bilateral anterior territory infarcts preoperatively, and the fourth patient had a small left anterior infarct (Fig 2). One patient (patient 5) had an acute infarct 3 months before surgery, which was not considered because no acute infarct was present on the immediate preoperative MR imaging. No posterior territory infarcts were observed before surgery. No control patient had a preoperative infarct.

To test whether the presence of an acute preoperative DWI lesion and/or impaired CVR correlated with the presence and location of postoperative infarcts, we evaluated 124 vascular regions in 31 patients ( 4 in each patient: left and right, anterior and posterior circulation). Of 8 anterior circulation territories with preoperative infarcts, 5 developed postoperative infarcts. Of 3 additional postoperative anterior circulation infarcts, 2 were contralateral to the preoperative DWI lesion. Overall, the presence of a preoperative DWI lesion was a strong predictor of postoperative infarct $(\mathrm{OR}=9.8 ; 95 \%$ confidence range, $2.2-44.2 ; P=.003$; Table 2).

\section{CBF and CVR Analysis}

Baseline anterior CBF showed a nonsignificant trend toward being lower in index patients (median, 36.6 versus $50.2 \mathrm{~mL} / 100$ $\mathrm{g} / \mathrm{min} ; P=.14$ ) but a significant difference in CBF following acetazolamide administration (median, 34.3 versus $63.7 \mathrm{~mL} /$ $100 \mathrm{~g} / \mathrm{min} ; P=.007)$. This finding corresponded to lower anterior circulation CVR in the index patients compared with controls (median, $-0.4 \%$ versus $26.3 \%$, respectively; $P=$ .003). There was no difference between groups for posterior circulation baseline CBF or CVR, in keeping with the known tendency of Moyamoya disease to preferentially involve the anterior circulation. In addition, within each hemisphere, there was significantly lower preoperative CVR in the regions with postoperative infarctions (Fig 3 ). Figure 4 shows an example of normal augmentation in a patient in the control group. Figure 5 highlights index patient 3 , with poor anterior augmentation, with additional evidence of reduced flow-related enhancement on an MR angiogram within the contralateral ICA following bypass, suggesting widespread hemodynamic changes following bypass.

\section{Univariate and Multivariate Analyses by Hemisphere}

Although preoperative DWI lesions showed the largest effect size as a predictor of postoperative infarct, impaired preoperative CVR (Fig 3) was also a highly consistent and significant risk factor for postoperative infarcts in univariate analysis by region (Table 2). This held true regardless of whether CVR was considered a continuous variable $(\mathrm{OR}=0.97 ; 95 \% \mathrm{CI}, 0.96-0.98 ; P<.001)$ or 


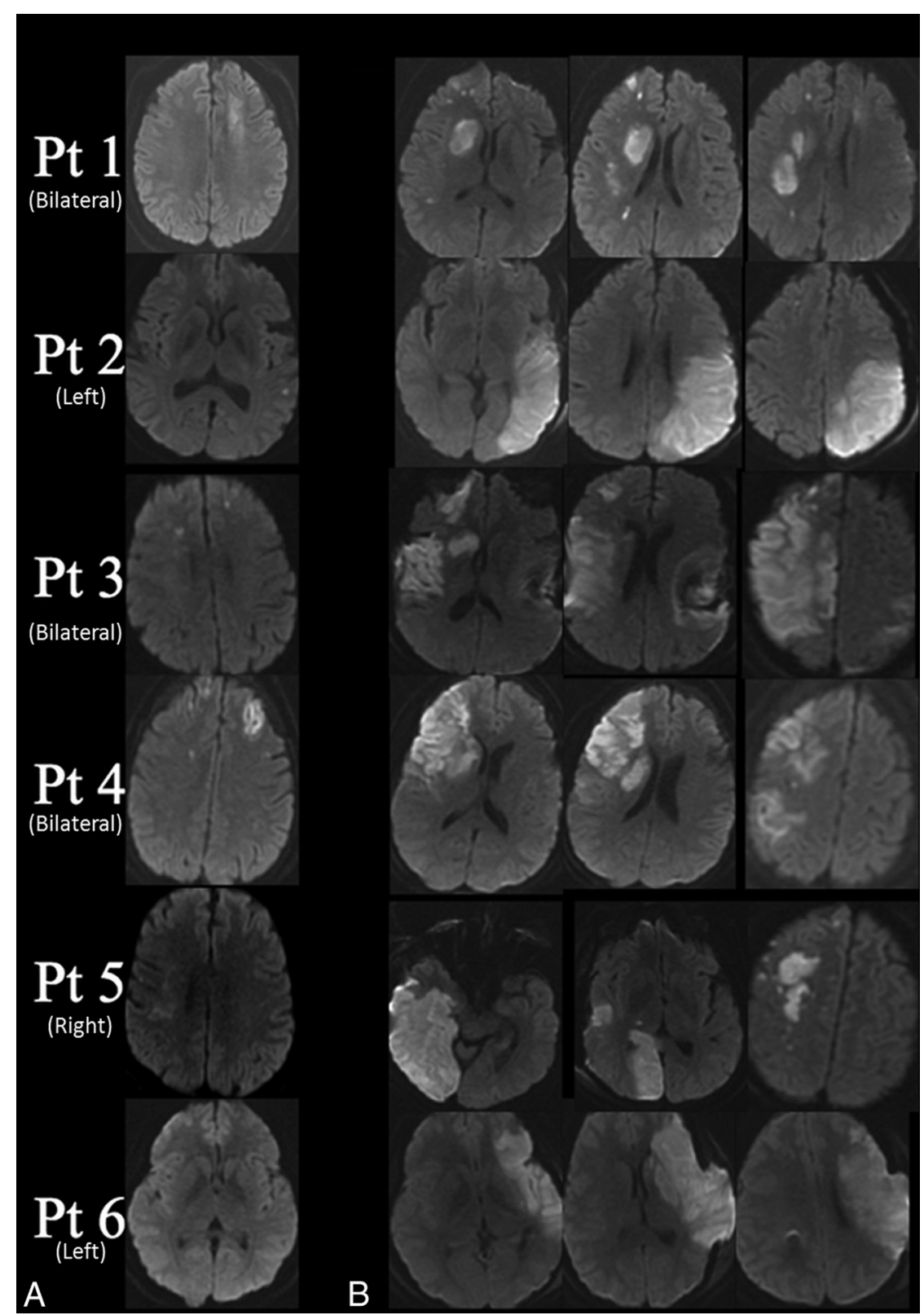

FIG 2. DWI in each index patient with the laterality of bypasses indicated in parentheses. $A$, Preoperative images demonstrate small acute infarcts in 4 of the 6 index patients. Patient 5 demonstrates a DWI region with positive findings in the right hemisphere that had negative findings on ADC (representing T2-shinethrough related to a subacute, rather than acute, infarct). $B$, Postoperative images demonstrate the extent of the infarct in the 6 index patients on MR imaging performed within 1 week of direct STA-MCA bypass.

\section{Table 2: Univariate predictors of large postoperative infarcts}

\begin{tabular}{|c|c|c|c|}
\hline Variable & $\begin{array}{l}\text { Odds } \\
\text { Ratio }\end{array}$ & $95 \% \mathrm{Cl}$ & $\begin{array}{c}P \\
\text { Value }\end{array}$ \\
\hline Presence of an infarct on preoperative MRI & 9.8 & $2.2-44.2$ & .003 \\
\hline Anterior location of preoperative infarct & 2.0 & $1.2-3.4$ & .01 \\
\hline Post-ACZ CBF augmentation (continuous, per $\mathrm{mL} / 100 \mathrm{~g} / \mathrm{min}$ ) & 0.97 & $0.96-0.98$ & $<.001$ \\
\hline Absolute CBF augmentation anterior circulation ( $10 \%$ change $)^{\mathrm{a}}$ & 0.88 & $0.82-0.96$ & .002 \\
\hline Impaired CVR (augmentation of $<10 \%$ ) & 2.8 & $1.8-4.3$ & $<.001$ \\
\hline Steal phenomenon (augmentation $<0 \%$ ) & 2.4 & $1.3-4.5$ & .005 \\
\hline
\end{tabular}

${ }^{a}$ The reported odds ratio is the effect of a $10 \mathrm{~mL} / 100 \mathrm{~g} / \mathrm{min}$ increase in absolute CBF change, a $10 \%$ increase in percentage difference for CBF change. For example, for absolute CBF change in the anterior circulation, for every increase of $10 \mathrm{~mL} / 100 \mathrm{~g} / \mathrm{min}$ (ie, better augmentation), there is a $12 \%$ decrease in the odds (ie, 0.88 ) that the patient will be in the index (ischemic complications) group. a binary variable, whether defined as $<10 \%$ increase $(\mathrm{OR}=2.8 ; 95 \% \mathrm{CI}, 1.8-$ $4.3 ; P<.001)$ or $<0 \%$ (steal) $(\mathrm{OR}=2.4$; 95\% CI, 1.3-4.5; $P=.005)$.

There were strong interactions between these predictors. For example, with the Wilcoxon rank sum analysis, the presence of a preoperative infarct strongly predicted impaired augmentation $(-12.7 \%$ versus $31.2 \%, P<.001)$. As such, we asked whether preoperative infarcts and impaired CVR may be independent risk factors. The results of multivariate generalized linear model regression adjusted for clustering are shown in Fig 6. Different probability fit curves depict the presence and absence of preoperative infarcts; the presence of preoperative infarct (red) shifts the curve up the probability axis relative to no preoperative infarct (blue). The best model incorporated the presence of a pre-bypass infarct and impaired CVR $(<10 \%$ increase) (Table 3$)$.

\section{DISCUSSION}

While the risk of developing a large infarct is very low following STA-MCA bypass, a small subset of patients do experience this complication shortly after the operation. Our results suggest that certain features evident on preoperative imaging may be related to this clinical outcome. While baseline CBF does not differ between the index and control groups, both univariate and multivariate regression analyses demonstrated that CVR is significantly lower in patients who develop large postoperative infarcts. Multiple prior studies suggest that reduced CVR is an independent risk factor for stroke. ${ }^{20,21}$ A recent meta-analysis demonstrated a significant positive relationship between reduced CVR and stroke risk in patients with carotid stenosis or occlusion, with an odds ratio of $3.86 .^{22}$ Despite this relationship, it is not immediately clear why patients would experience strokes so soon after revascularization.

One potential explanation for the observed infarcts is that any surgical procedure represents a physiologic stress that portends an elevated risk of ischemic complications. Indeed, for this reason, a cardiac stress test is routinely performed in patients considered at high risk before surgical intervention. 


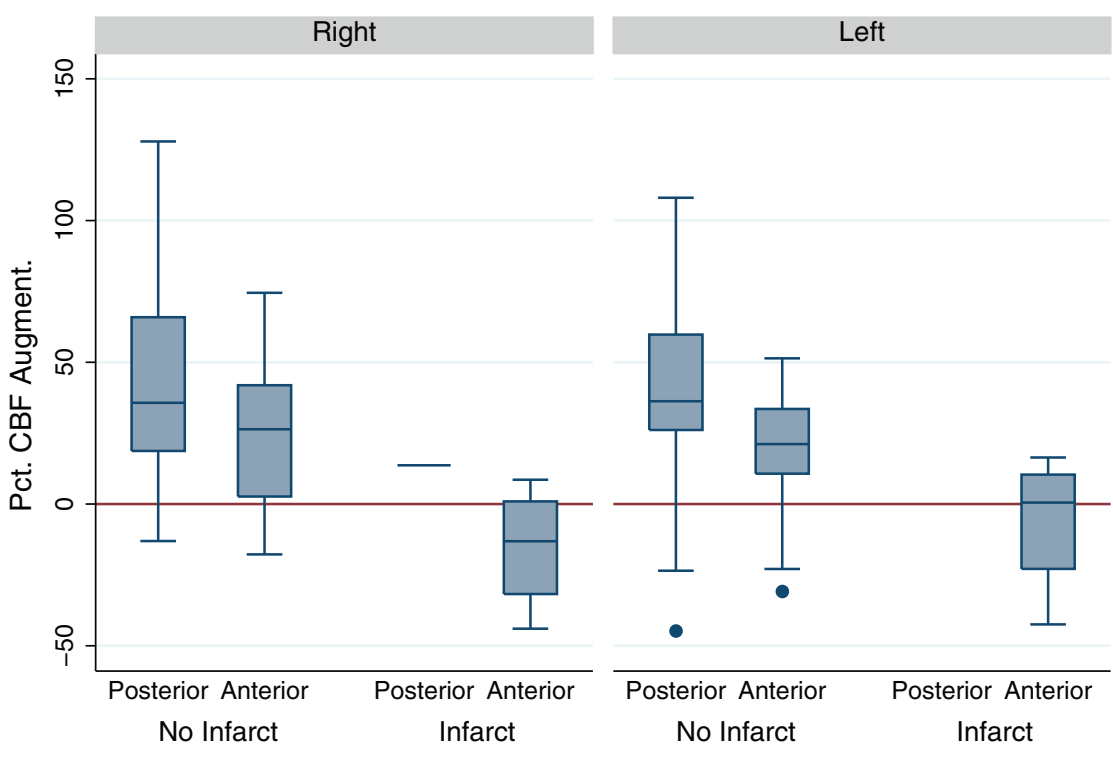

Graphs by Hemisphere

FIG 3. Cerebrovascular reserve expressed as percentage change from baseline within the right and left anterior and posterior circulation ROls, segregated by the presence or absence of postoperative infarcts. All postoperative infarcts were in the anterior circulation, with the exception of 1 patient who developed a posterior circulation infarct on the side of a fetal posterior cerebral artery. CVR was lower in the hemispheres that subsequently developed infarcts, compared with those that did not. There was no difference between hemispheres. Circles indicate individual outlier measurements.

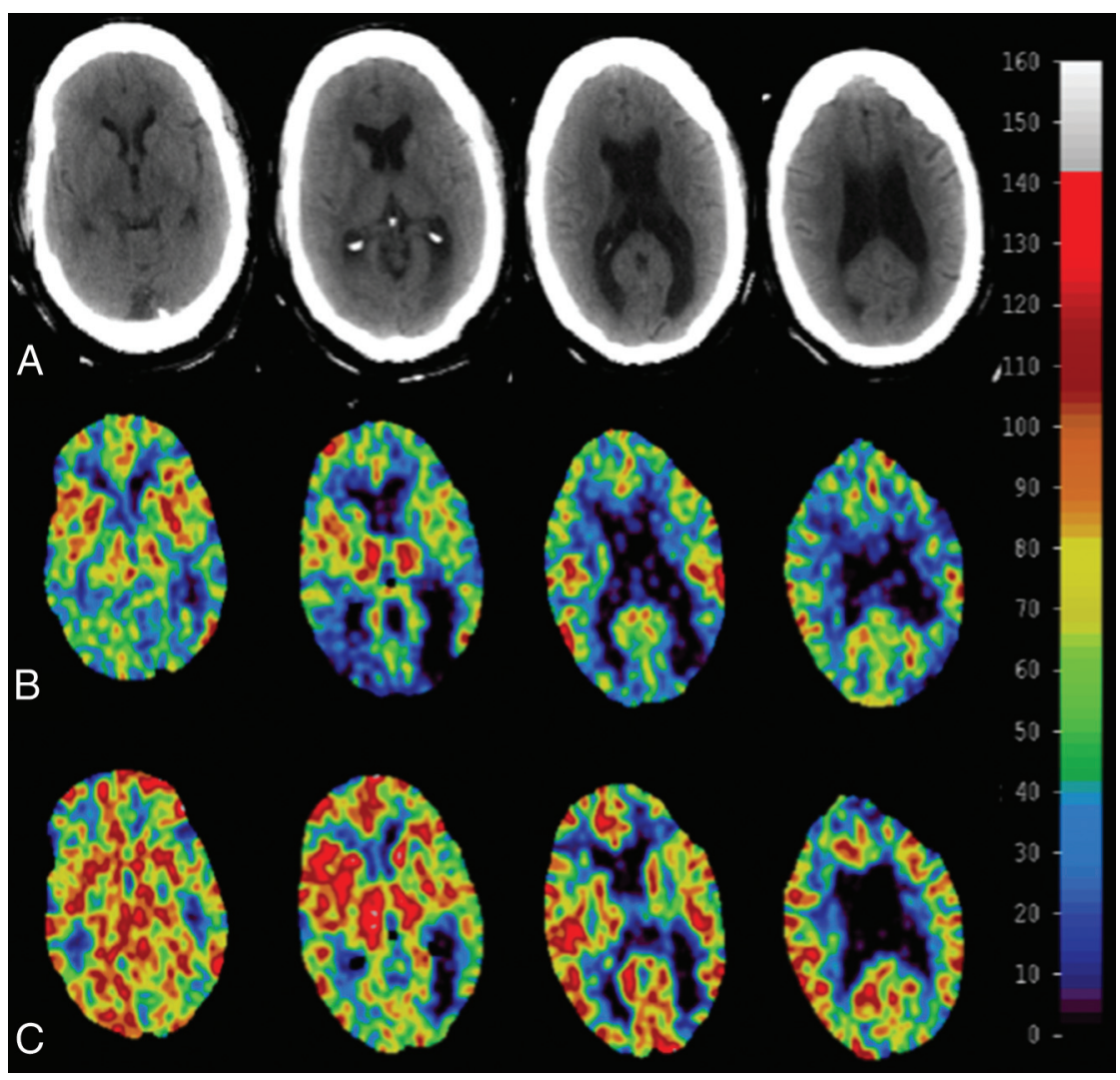

FIG 4. In this representative control patient, quantitative XeCT CBF maps (scale at right in milliliters $/ 100 \mathrm{~g} / \mathrm{min}$ ) at 4 representative axial levels $(A)$ obtained before $(B)$ and after $(C)$ the administration of acetazolamide are shown. CVR, calculated from these images, through the Equation referenced in the "Materials and Methods" section, is within the normal expected range (>30\%) in all vascular territories, and this patient had no postoperative infarcts or clinical symptoms.
Similarly, patients with reduced CVR may be at an elevated risk of ischemic complications following any operation. Schoof et $\mathrm{al}^{23}$ explored this concept in patients who underwent a coronary artery bypass graft and were screened with carotid sonography. If stenosis was identified, CVR was calculated by using transcranial Doppler before and after carbon dioxide administration. Patients with reduced CVR had a $27.3 \%$ stroke rate following cardiothoracic surgery, compared with a $1.5 \%$ risk in patients with normal CVR. Patients with reduced CVR may similarly have an elevated stroke risk following cerebral revascularization.

The rationale behind operative intervention in patients with Moyamoya disease is that for the long term, the risk of ischemic events will be lowered. As such, patients with reduced CVR may benefit the most. Indeed, the presence of reduced CVR appears to promote the success of revascularization. In a study assessing the postoperative course of patients with Moyamoya disease who underwent bilateral encephaloduroarteriosynangiosis, revascularization occurred selectively in areas of reduced CVR, ${ }^{24}$ suggesting that abnormal CVR may itself promote angiogenesis. With time, this possibility is obviously advantageous. However, during an operation or in the immediate postoperative period, during which mean flow through the microanastomosis increases 5-fold (from 4.4 to $22.2 \mathrm{~mL} / \mathrm{min}$ ), ${ }^{9}$ the balance of cerebral hemodynamics may be very fragile. Increased flow related to the bypass may paradoxically represent a new hemodynamic stress and induce altered perfusion parameters by competing with the underlying stenotic vasculature and the native collateral network. This concept has been explored in prior studies, in part to account for the presence of postoperative perfusion abnormalities contralateral to the bypass. ${ }^{1,6}$ Indeed, it has previously been shown that increased STA flow following bypass is correlated with a higher incidence of postoperative infarct, ${ }^{9}$ though it is not clear whether this association is causative or correlative.

As in our study, infarcts have been reported both ipsilateral and contralateral to the most recently revascularized 


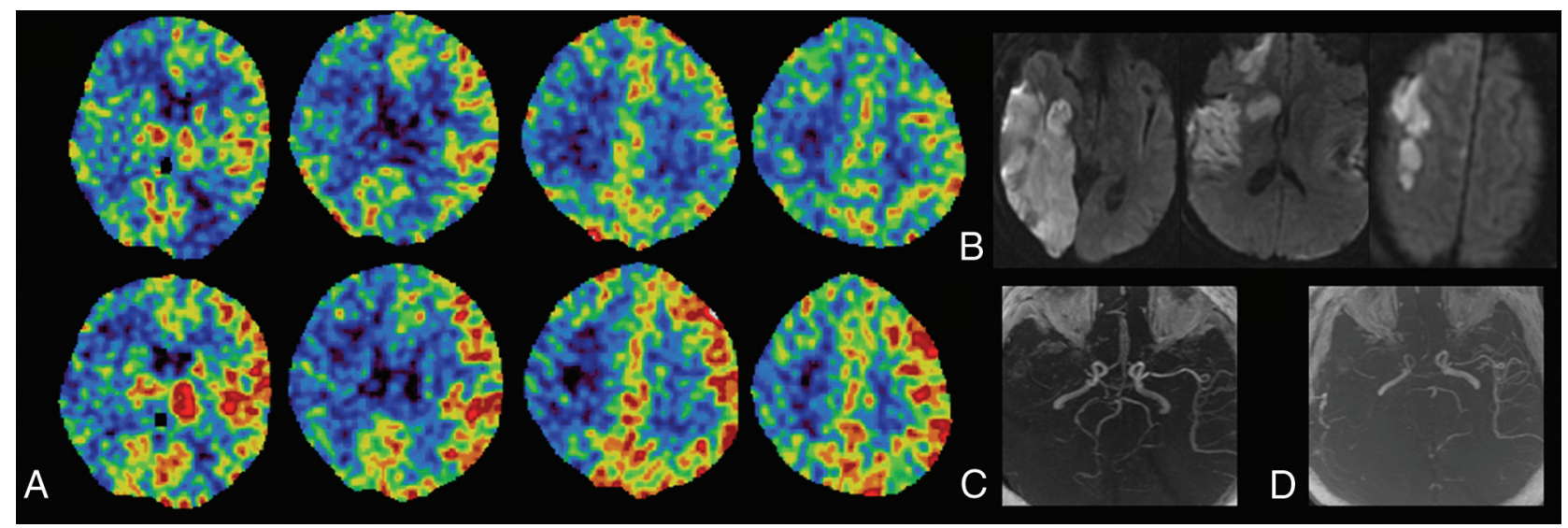

FIG 5. A 51-year-old man with Moyamoya disease and a history of transient left arm numbness and left lower extremity weakness (patient 3 ). $A$, $\mathrm{XeCT}$ imaging demonstrates mildly reduced baseline CBF in the right anterior circulation (top row). Postacetazolamide CBF paradoxically decreases in the right anterior circulation (bottom row), representing steal physiology, while augmenting normally on the left. Following right STA-MCA bypass, he experienced progressive left hemiplegia. B, Postoperative MR imaging within the first week after bypass revealed an extensive new infarct involving the right MCA territory and a portion of the right PCA territory. We believe that the posterior circulation involvement was due to the presence of a large posterior communicating artery on this side. C, Preoperative MR angiography reveals severe steno-occlusive disease involving the right-greater-than-left supraclinoid ICAs with complete occlusion of the right M1 MCA segment. D, MRA obtained after revascularization demonstrates a patent right STA-MCA bypass but decreased conspicuity of the patient's native vasculature, despite the study being performed on the same scanner. In particular, there is no appreciable flow-related enhancement in the bilateral anterior cerebral arteries or in the right posterior communicating or posterior cerebral artery, and there is continued occlusion of the right MCA. While the changes seen could be multifactorial and could be related to changes in other factors, such as sedation or intracranial pressure, we suggest that the hemodynamic changes related to the operation may have caused worsening of the patient's tenuous native right-sided arterial circulation, particularly given the lack of significant changes on the left side.

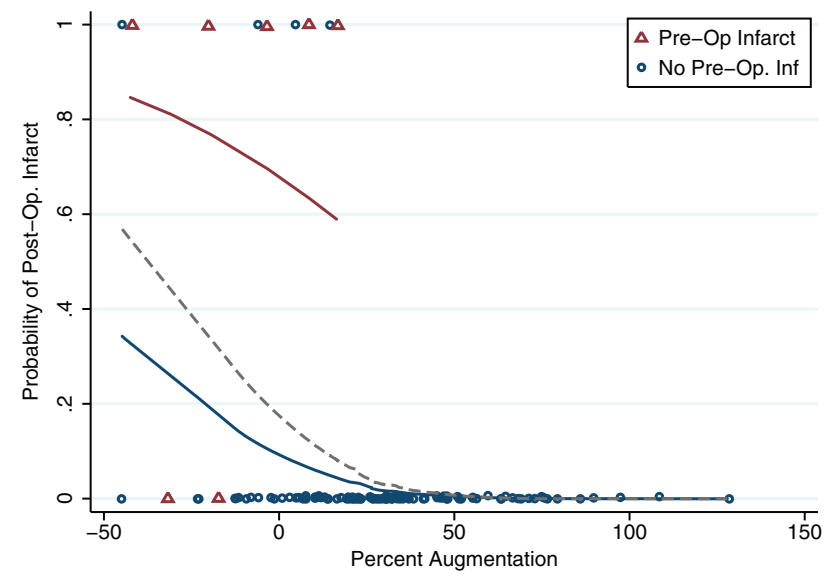

FIG 6. Probability fit curves based on multivariate analysis. Findings illustrate that impaired augmentation strongly increases the probability of infarct both in the presence (red line) and absence (blue line) of a preoperative infarct. The dashed line shows the overall probability of infarct based on percentage augmentation, regardless of preoperative infarct.

Table 3: Multivariate analysis summary

\begin{tabular}{|c|c|c|c|}
\hline Variable & $\begin{array}{l}\text { Odds } \\
\text { Ratio }\end{array}$ & $95 \% \mathrm{Cl}$ & $\begin{array}{c}P \\
\text { Value }\end{array}$ \\
\hline Prese & 6.9 & $1.3-36.1$ & .02 \\
\hline An & 1.1 & $0.7-1.8$ & 632 \\
\hline Impaired CVR (CBF augmentation $<10 \%$ ) & 2.0 & $1.4-3.0$ & $<.001$ \\
\hline
\end{tabular}

Note:-preop indicates preoperative.

hemisphere. The concept of competitive flow might further explain the prominent changes in vascular flow dynamics following bypass in several of the index cases. For instance, a postoperative MR angiogram obtained in 1 patient (Fig 5) demonstrated new decreased flow-related enhancement in the bilateral anterior and right posterior communicating/posterior cerebral artery territories, which was confirmed as a change on cerebral angiography. It is possible that such rapid progression could arise in response to hemodynamic changes related to the flow through the new bypass in conjunction with altered perfusion parameters in the setting of a large acute infarct.

Most of the index cases demonstrated small acute infarcts on MR imaging examinations performed shortly before the operation. On univariate and multivariate analyses, the presence of a preoperative acute infarct was found to be a significant and independent predictor of infarct in the postoperative period. Most interesting, although the DWI lesions predicted the vascular territory in 5 of 8 anterior circulation territories, 2 anterior circulation infarcts occurred contralateral to the preoperative DWI lesion. These occurred in patients with large infarcts after the first bypass but who had significant contralateral stenosis and poor CVR. While such spatially removed infarcts may be explained by the hemodynamic changes described above, systemic factors (such as alterations in platelet function) may render a patient with recent infarcts more generally susceptible to a postoperative infarct than would be expected on the basis of vascular pathology alone. In addition, while hypotension was strictly avoided during the operation, other operative stresses, such as cortisol release and platelet activation, may collectively contribute to perioperative ischemia. Whether delaying operative revascularization to allow infarct evolution/stabilization in such high-risk patients would have altered the postoperative course is presently unknown because our data were collected as part of the preoperative work-up of operative patients and similar data were not available to assess the effect of acute infarct and impaired CVR on a matched nonoperative patient cohort. Nonetheless, the risks 
and benefits of delaying the operation in patients with ongoing ischemic change must be carefully weighed, given the inherently progressive nature of Moyamoya disease.

Prior researchers have noted the association between preoperative infarct and perioperative complications in Moyamoya disease. In a review of 165 adult patients (undergoing 265 revascularization procedures), Hyun et $\mathrm{al}^{25}$ found that the presence of a preoperative diffusion abnormality was significantly correlated with 19 perioperative infarcts. However, most of these complications were transient in nature and occurred primarily in indirect revascularization procedures. By comparison, our series assesses variables associated with large, clinically significant infarctions in patients undergoing direct bypass, a distinctly less well-studied patient population.

There are several potential limitations to our study. The first is its retrospective design and the relatively small number of index cases. Our institutional morbidity associated with cerebral revascularization is low, and despite a large number of bypasses, only a small number of patients with large postoperative infarcts were available for analysis. To maximize the power of this small patient cohort, we analyzed multiple vascular territories in each patient independently, while correcting for clustering. We also performed multivariate analysis, maximizing our ability to detect meaningful predictors of postoperative infarct. Second, this analysis focused on 2 smaller subsets of a larger group of patients with Moyamoya disease. The study was specifically designed in this manner to identify factors associated with profound operative morbidity relative to the optimal clinical outcome.

While prior studies have assessed factors associated with transient postoperative neurologic deficits, ${ }^{26}$ our index patients represent a small, relatively unstudied subgroup. Given the low risk of postoperative stroke in this patient population and the fact that most of these strokes tend to be small, investigation of the factors that lead to severe infarcts was deemed most clinically relevant. Nonetheless, additional patients who underwent bypass at our institution included those with either small postoperative diffusion abnormalities (often clinically asymptomatic) or those with mild or transient neurologic symptoms. Further studies are ongoing to address contributors to adverse outcomes in these larger patient cohorts. Only acute (DWI with positive findings) preoperative infarcts were considered in the evaluation of preoperative risk factors because only acute infarcts could be consistently evaluated in all patients. Although 4 of the 6 index cases had acute preoperative infarcts, another index patient had a small infarct on a 3-month preoperative scan, which was subacute on immediate preoperative MR imaging. Although the presence of subacute infarcts could not be consistently evaluated for all patients in this study, we cannot exclude the possibility that the presence of subacute infarcts could also portend a worse prognosis.

Finally, we used XeCT CBF measurements for the CVR measurements. While XeCT is considered a reference standard method, others have proposed the use of MR imaging-based perfusion measurements. ${ }^{27}$ Regardless of the methodology, our results underscore the importance of quantitative assessment of CVR to identify patients at high risk for stroke. Thus, we are now investigating the use of MR perfusion with and without acetazolamide, which, if comparable, may represent a technique more amenable to widespread use outside specialized centers.

\section{CONCLUSIONS}

Our results indicate that an acute infarct on preoperative MR imaging and the presence of impaired CVR can help identify the small subset of patients with Moyamoya who experience a severe ischemic complication following extracranial-intracranial bypass.

Disclosures: Gary K. Steinberg—UNRELATED: Consultancy: Medtronic Neuroscience Strategic Advisory Board; Grants/Grants Pending: National Institutes of Health, ${ }^{*}$ California Institute for Regenerative Medicine*; Patents (planned, pending or issued): Stanford University*; Stock/Stock Options: Qool Therapeutics. Greg Zaharchuk-RELATED: Grant: National Institutes of Health,* Comments: part of a larger study of patients with Moyamoya disease, 5R01-NS066506; UNRELATED: Consultancy: GE Healthcare; Grants/Grants Pending: National Institutes of Health,* Comments: multiple grants unrelated to the current work; Royalties: Cambridge University Press. *Money paid to the institution.

\section{REFERENCES}

1. Ma Y, Meng L, Jiao LQ, et al. Contralateral cerebral hemodynamic changes after unilateral direct revascularization in patients with Moyamoya disease. Neurosurg Rev 2011;34:347-54 CrossRef Medline

2. Ikezaki K. Rational approach to treatment of Moyamoya disease in childhood. J Child Neurol 2000;15:350-56 CrossRef Medline

3. Togao O, Mihara F, Yoshiura T, et al. Cerebral hemodynamics in Moyamoya disease: correlation between perfusion-weighted MR imaging and cerebral angiography. AJNR Am J Neuroradiol 2006;27: 391-97 Medline

4. Suzuki J, Takaku A. Cerebrovascular "Moyamoya" disease: disease showing abnormal net-like vessels in base of brain. Arch Neurol 1969;20:288-99 CrossRef Medline

5. Yun TJ, Cheon JE, Na DG, et al. Childhood Moyamoya disease: quantitative evaluation of perfusion MR imaging - correlation with clinical outcome after revascularization surgery. Radiology 2009;251:216-23 CrossRef Medline

6. Pandey $P$, Steinberg GK. Neurosurgical advances in the treatment of Moyamoya disease. Stroke 2011;42:3304-10 CrossRef Medline

7. Karasawa J, Kikuchi H, Furuse S, et al. Treatment of Moyamoya disease with STA-MCA anastomosis. J Neurosurg 1978;49:679-88 CrossRef Medline

8. Guzman R, Lee M, Achrol A, et al. Clinical outcome after 450 revascularization procedures for Moyamoya disease: clinical article. J Neurosurg 2009;111:927-35 CrossRef Medline

9. Lee M, Guzman R, Bell-Stephens T, et al. Intraoperative blood flow analysis of direct revascularization procedures in patients with Moyamoya disease. J Cereb Blood Flow Metab 2011;31:262-74 CrossRef Medline

10. Powers WJ, Clarke WR, Grubb RL Jr, et al; COSS Investigators. Extracranial-intracranial bypass surgery for stroke prevention in hemodynamic cerebral ischemia: the Carotid Occlusion Surgery Study randomized trial. JAMA 2011;306:1983-92 CrossRef Medline

11. EC/IC Bypass Study Group. Failure of extracranial-intracranial arterial bypass to reduce the risk of ischemic stroke: results of an international randomized trial. N Engl J Med 1985;313:1191-200 CrossRef Medline

12. Straka M, Albers GW, Bammer R. Real-time diffusion-perfusion mismatch analysis in acute stroke. J Magn Reson Imaging 2010;32: 1024-37 CrossRef Medline

13. Qiu D, Straka M, Zun Z, et al. CBF measurements using multidelay pseudocontinuous and velocity-selective arterial spin label- 
ing in patients with long arterial transit delays: comparison with xenon CT CBF. J Magn Reson Imaging 2012;36:110-19 CrossRef Medline

14. Zaharchuk G, Bammer R, Straka M, et al. Improving dynamic susceptibility contrast MRI measurement of quantitative cerebral blood flow using corrections for partial volume and nonlinear contrast relaxivity: a xenon computed tomographic comparative study. J Magn Reson Imaging 2009;30:743-52 CrossRef Medline

15. Settakis G, Molnar C, Kerenyi L, et al. Acetazolamide as a vasodilatory stimulus in cerebrovascular disease and in conditions affecting the cerebral vasculature. Eur J Neurol 2003;10:609-20 CrossRef Medline

16. Chang SD, Steinberg GK. Surgical management of Moyamoya disease. Moyamoya.com. http://www.moyamoya.com/journals/ moyamoya.html. Last update January 1, 2013. Accessed April 1, 2015

17. Li G, Lim M, Khan N, et al. Cerebral revascularization for Moyamoya disease. In: Abdulrauf SI, ed. Cerebral Revascularization: Techniques in Extracranial-to-Intracranial Bypass Surgery. Philadelphia: Elsevier; 2011:185-92

18. Guzman R, Steinberg GK. Direct bypass technique for the treatment of pediatric Moyamoya disease. Neurosurg Clin N Am 2010;21: 565-73 CrossRef Medline

19. JET Study Group. Japanese EC-IC bypass trial (JET study): study design and interim analysis [in Japanese]. Surg Cereb Stroke (Jpn) 2002;30:97-100 CrossRef

20. Kuroda S, Houkin K, Kamiyama H, et al. Long-term prognosis of medically treated patients with internal carotid or middle cerebral artery occlusion: can acetazolamide test predict it? Stroke 2001;32: 2110-16 CrossRef Medline

21. Grubb RL Jr, Derdeyn CP, Fritsch SM, et al. Importance of hemodynamic factors in the prognosis of symptomatic carotid occlusion. JAMA 1998;280:1055-60 CrossRef Medline

22. Gupta A, Chazen JL, Hartman M, et al. Cerebrovascular reserve and stroke risk in patients with carotid stenosis or occlusion: a systematic review and meta-analysis. Stroke 2012;43:2884-91 CrossRef Medline

23. Schoof J, Lubahn W, Baeumer M, et al. Impaired cerebral autoregulation distal to carotid stenosis/occlusion is associated with increased risk of stroke at cardiac surgery with cardiopulmonary bypass. J Thorac Cardiovasc Surg 2007;134:690-96 CrossRef Medline

24. Nariai T, Suzuki R, Matsushima Y, et al. Surgically induced angiogenesis to compensate for hemodynamic cerebral ischemia. Stroke 1994;25:1014-21 CrossRef Medline

25. Hyun SJ, Kim JS, Hong SC. Prognostic factors associated with perioperative ischemic complications in adult-onset Moyamoya disease. Acta Neurochir (Wien) 2010;152:1181-88 CrossRef Medline

26. Ohue S, Kumon Y, Kohno K, et al. Postoperative temporary neurological deficits in adults with Moyamoya disease. Surg Neurol 2008; 69:281-86; discussion 286-87 CrossRef Medline

27. Tanaka Y, Nariai T, Nagaoka T, et al. Quantitative evaluation of cerebral hemodynamics in patients with Moyamoya disease by dynamic susceptibility contrast magnetic resonance imaging: comparison with positron emission tomography. J Cereb Blood Flow Metab 2006;26:291-300 CrossRef Medline 